\title{
Interdisziplinäre Herausforderungen der Karsthydrogeologie
}

\author{
Nico Goldscheider • Tobias Geyer
}

Online veröffentlicht: 5.11.2013

(C) Springer-Verlag Berlin Heidelberg 2013

Karstgrundwasserleiter sind in vielen Regionen der Welt wertvolle Trinkwasserressourcen, aber gleichzeitig aufgrund ihrer speziellen Struktur und Eigenschaften besonders verletzlich gegenüber Schadstoffeinträgen und oft schwierig zu erkunden und zu bewirtschaften. Daraus ergeben sich vielfältige praktische Fragestellungen und wissenschaftliche Herausforderungen für die Karsthydrogeologie sowie ein ganz eigenes Instrumentarium an Methoden und Modellansätzen, von denen einige in diesem und dem folgenden Themenheft präsentiert werden.

Karstsysteme sind weit mehr als Wasserressourcen. Sie sind Ökosysteme, Inseln der Biodiversität, natürliche Senken für $\mathrm{CO}_{2}$, Klimaarchive und mehr. An der Oberfläche bieten sie vielfältige und einzigartige Lebensräume für Pflanzen und Tiere, darunter zahlreiche endemische Arten bis hin zum hochgradig bedrohten Delacour's Langur, einer Primatenart, die ausschließlich in einigen vietnamesischen Karstgebieten anzutreffen ist. Auch unterirdisch und im Grundwasser beherbergen Karstsysteme viele endemische Tierarten, darunter Wirbellose, Fische und Amphibien, wie den Höhlensalamander Proteus anguinus. Hieraus ergeben sich spannende interdisziplinäre Forschungsfragen zwischen Karsthydrogeologie und Biologie.

Böden in Karstlandschaften bilden die Grundlage für natürliche Vegetation und landwirtschaftliche Produktion, sind

N. Goldscheider $(\otimes)$

Institut für Angewandte Geowissenschaften,

KIT - Karlsruher Institut für Technologie,

Kaiserstr. 12, 76131 Karlsruhe, Deutschland

E-Mail: nico.goldscheider@kit.edu

T. Geyer

Landesamt für Geologie, Rohstoffe und Bergbau

im Regierungspräsidium Freiburg,

Albertstr. 5, 79104 Freiburg, Deutschland

E-Mail: tobias.geyer@rpf.bwl.de aber besonders anfällig gegenüber Erosion. Ist der Boden einmal verschwunden, bleibt nur nackter Kalkstein übrig. Eine Neubildung ist in menschlichen Zeithorizonten kaum möglich. Erodierte Bodenpartikel werden in den Untergrund eingespült und bilden dort Höhlensedimente. Mit dem Verlust des Bodens verschwindet eine wichtige Schutzschicht gegenüber Schadstoffeinträgen ins Grundwasser. Der Verkarstungsprozess, also die Lösung von Karbonat-Mineralen, stellt eine natürliche Senke für atmosphärisches $\mathrm{CO}_{2}$ dar und wirkt daher dem Klimawandel entgegen. Dieser Prozess ist unter Bodenbedeckung effektiver als auf nacktem Kalkstein, weil im Boden durch mikrobiellen Abbau abgestorbener Pflanzenreste höhere $\mathrm{CO}_{2}$-Partialdrücke auftreten als in der Atmosphäre. Bodenerosion verringert also die Wirksamkeit des Karstprozesses als $\mathrm{CO}_{2}$-Senke. Die skizzierten Zusammenhänge verdeutlichen das Potenzial bzw. den Bedarf an interdisziplinärer Forschung zwischen Bodenkunde, Agrarwissenschaft, Mikrobiologie, Klimaforschung und Karsthydrogeologie.

Stalagmiten aus Karsthöhlen werden zunehmend als Klimaarchive genutzt und sind als solche den Eisbohrkernen in mancher Hinsicht überlegen, denn Stalagmiten findet man in fast allen Höhlen, überall auf der Welt, in allen Klimazonen, während brauchbare Eisbohrkerne nur in Grönland und der Antarktis gewonnen werden können - mit größtem technischem Aufwand. Paläoklimatische Daten aus Stalagmiten sind nur dann vernünftig interpretierbar, wenn die hydrologische Funktion der ungesättigten Zone oberhalb der betrachteten Höhle besser verstanden wird - eine weitere interdisziplinäre Herausforderung für die Karsthydrogeologie.

Aufgrund der skizzierten vielfältigen Zusammenhänge zwischen Wasser, Böden, Biodiversität und Klima sollte die zukünftige Karstforschung wesentlich interdisziplinärer ausgerichtet sein als dies heute der Fall ist, wobei dem Grundwasser - und damit auch der Hydrogeologie - bei allen genannten Aspekten eine wichtige Rolle zukommt. 